\title{
The Use of Ethyl Cellulose Polymer to Control Drug Release of Hydrocortisone Acetate
}

\author{
MAHDIE TORKAMAN ${ }^{1}$ and FATEMEH ZARE KAZEMABADI ${ }^{1,2 *}$ \\ 'Department of Chemical Engineering, Science and Research Branch, Islamic \\ Azad University, Tehran, Iran. \\ ${ }^{2}$ Young Researchers and Elites club, Science and Research Branch, Islamic \\ Azad University, Tehran, Iran. \\ ${ }^{*}$ Corresponding author E-mail: fatemeh.zarekazemabadi@chmail.ir
}

http://dx.doi.org/10.13005/ojc/330444

(Received: August 12, 2017; Accepted: December 12, 2017)

\begin{abstract}
There are considerable numbers of medicines that cannot be dissolved in water or have a little aqueous solubility, and this causes problems in their absorption and reduction of bioavailability of them subsequently. A variety of methods have been developed to increase the solubility of drugs to treat these problems. In this study, ethyl cellulose polymers have been used to deliver hydrocortisone acetate drugs. In this way, solvent evaporation technique was considered to increase the solubility of the drug hydrocortisone acetate. Scanning electron microscopy (SEM) was used to determine the shape and size of the microcapsules. The infrared spectroscopy (FT-IR) was also applied to investigate that if any change was happened in the structure of drug during the microcapsulation process.
\end{abstract}

Keywords: ethyl cellulose, solvent evaporation, hydrocortisone acetate, microcapsules, drug delivery.

\section{INTRODUCTION}

Today, due to population growth and increase of various diseases, use of drugs is one of the world's major issues. On the other hand, undesirable side effects of some medications and high prices, makes the field of study using the least amount of medication with the highest efficiency. Many drugs have side effects caused by improper use. Thus, drug makers have tried that by careful control of the drug concentration in the blood, minimize these side effects ${ }^{1}$. The aim of any delivery system is to strengthen or facilitate the therapeutic effect. Ideally, drug delivery systems can be used to maximize the desired therapeutic response and correct the amount of medicine given to the effect on the speed and timing of release ${ }^{2}$. These systems defined to deliver drugs at a predetermined for predetermined rate and periods, to overcome the shortage of conventional drug formulations ${ }^{3}$. Different 
drugs, based on the solubility and absorption condition, are taken by different concentrations. If the rate of drug absorption is low, it raises consumption that will also increase side effects. Drug delivery technology advantages compared to traditional medicine (drug) is that improves efficiency, reduces toxicity, patients feel improvement becomes more pronounced in easier ways. This time may take from days to months, and it may take years ${ }^{4}$.

\section{Hydrocortisone Acetate}

Drugs are categorized in different types based on their solubility including instant soluble, soluble, slightly soluble, very low soluble, insoluble and so forth. Corticosteroids are a group of drugs widely used in medicine as an anti-inflammatory and anti-allergic agent. Hydrocortisone acetate (HCA) is a member of corticosteroids. Hydrocortisone acetate injection form dosage is frequently used for the treatment of many different conditions such as allergic disorders and respiratory or skin diseases. Reports on quantitative analysis of hydrocortisone acetate in pharmaceutical formulations, especially in injectable liquid form, are relatively sparse. The first report about analysis of the Hydrocortisone Acetate Products business goes back to 1975 . Increase of the solubility of drugs includes a variety of techniques such as microcapsulation. Microcapsulation is a process of putting the material into a polymer capsule in micro or nano size ${ }^{5}$. Solvent evaporation is one of the methods frequently used in encapsulation of the drugs. Many factors affect the quality and size of the capsules. All the parameters must be controlled to produce a desired product. Otherwise, the slightest change in any of these parameters is very effective in size and quality aspects of microencapsulation. Therefore, in the process we should take into consideration the engineering parameters and regulate the production system by adjusting these parameters. Because of high efficiency, as this method is easy and does not require advanced equipment, today solvent evaporation is considered as a desirable method to increase the solubility of drugs $^{6}$. The aim of this experiment is to study the changes of solubility and to determine the solubility of drug delivery e after microcapsulation processes.

\section{Theoretical basis}

During the twentieth century, research were conducted on the development of materials for controlled drug release, including use of liposomes for targeted active and inactive areas of infectious disease and cancer, locally advanced biodegradable polymers to deliver bioactive agents, water-soluble polymers to increase the half-life of therapeutic proteins and decreased immune response, linear polymer chains the entire drug, and micellar nanoparticles to encapsulate Sahtarhay, macromolecules such as this study is to deliver nucleic acids $^{7}$. In addition, the lipid formulations that produce solid particles (or semi-solid) after subcutaneous injection, are created as implants. Delivery systems are attractive for preantral applications. The use of this system in comparison with implants that require local anesthesia and anesthesia are less aggressive and on the other hand, it can give the prolong topical drug delivery for periods of time ${ }^{8}$. Case of controlled release of the drug from the microspheres was studied since 1970 and it was clinically available for first time in the late '80s. Langer and Folkman were the pioneers in this field who demonstrated that proteins could release from biodegradable polymer matrices. One of the techniques has attracted much attention about obtaining long-term drug delivery is about the compilation of drug in the core of microencapsules. Outer polymeric covering of these injectable particles acts as a membrane tool that controls the rate of drug release from the core of les, a covering the solid particles of the drug, aqueous solutions can also be encapsulated. Change (1976) studied the microcapsulaton of proteins such as enzymes, hormones and vaccines. Biodegradable microcapsules cross connected of albumin were described by Benita et al to treat blockages in blood flow instead of drug delivery. Also applications of microcapsulation was revised by Salib (1977) 9 . In the process of solvent evaporation, hardening of emulsion also occurs. Using a vacuum or mild increase, we can accelerated solvent evaporation. In the process of solvent extraction, emulsion is transferred into large quantities of water or other cooling medium and organic solvent extraction occurs faster than other methods. Therefore, microspheres prepared by solvent extraction methods are more porous than those prepared by solvent evaporation. Usually the porous structure causes faster release of the proteinpeptide drugs. Finally, prepared microspheres are collected by centrifugation or filtration and freeze drying $^{10}$. 


\section{MATERIALS AND METHODS}

In this study, hydrocortisone acetate was used in order to increase the solubility of poor drug release. Preparation of microcapsules of hydrocortisone acetate and their characterization was done by ethyl cellulose polymer. Microcapsules containing hydrocortisone acetate were prepared and optimized using solvent evaporation technique. Evaluation and comparison of the pure drug and microcapsules solubility in water was also taken. The microencapsulates will also be used to study drug release in aqueous environments beds as simulators of $\mathrm{pH}$ of the stomach and body. Required materials and solutions: 1 . hydrocortisone acetate, 2-ethyl cellulose, 3-polyvinyl alcohol (PVA), 4-dichloromethane, 5-distilled water, 6- $\mathrm{HCl}$, 7-chloroform, 8-salts sodium phosphate: $\mathrm{Na}_{2} \mathrm{HPO}_{4}$, $\mathrm{NaH}_{2} \mathrm{PO}_{4}$.

\section{Production of formulations}

Preparation of Hydrocortisone Acetate drug microcapsules was done by solvent evaporation technique. In this way, $0.06 \mathrm{~g}$ of hydrocortisone acetate with $0.35 \mathrm{~g}$ of ethyl cellulose was dissolved in a volume of $10 \mathrm{ml}$ dichloromethane solvent. This solution acts as an internal phase. External phase was prepared by dissolving $0.5 \mathrm{~g}$ of polyvinyl alcohol in $150 \mathrm{ml}$ of distilled water. Then the internal phase is added drop by drop to the stirring external phase. The resulting mixture is placed on the magnetic stirrer for $18 \mathrm{~h}$ to completely vaporize the organic solvent. Obtained microcapsules are separated by the filter paper and dried for $24 \mathrm{~h}$ at room temperature.

\section{Test method}

A certain amount of produced microcapsules of $0.048 \mathrm{~g}$ were dissolved in $10 \mathrm{ml}$ of chloroform. The amount of hydrocortisone acetate in them was determined by UV-Vis spectrophotometer at a wavelength of $290 \mathrm{~nm}$ is determined. Values of $0.001,0.002,0.003, .004$ and 0.005 grams of pure hydrocortisone acetate drug were separately dissolved in a volume of $10 \mathrm{ml}$ of chloroform and hydrocortisone acetate value in them was characterized by spectrophotometry UV- Vis at a wavelength of $290 \mathrm{~nm}$. Furthermore in order to ensure that microcapsulation process did not change the nature of the drug hydrocortisone acetate, the microcapsules were studied by infrared spectrophotometer (FT-IR). Values of all materials used included of hydrocortisone acetate, ethyl cellulose, dichloromethane and polyvinyl alcohol were omptimized in continue. Then drug solubility was studied in order to study the effect of microcapsulation process on increase of the solubility of poorly soluble hydrocortisone acetate drugs in water. In this way, $0.1 \mathrm{~g}$ of the net hydrocortisone acetate drug, the $0.26 \mathrm{~g}$ of microcapsules containing drugs and $0.19 \mathrm{~g}$ of drug-free microcapsules were weighed and poured in $20 \mathrm{ml}$ of distilled water separately. The resulting mixtures were placed in incubator shaker for $24 \mathrm{~h}$ at $37^{\circ} \mathrm{C}$ and during this period at 1, 4, 8 and $24 \mathrm{~h}, 2 \mathrm{ml}$ of samples were removed and the amount of hydrocortisone acetate in them was detected by UV-Vis spectrophotometer at a wavelength of $290 \mathrm{~nm}$. Then the body conditions such as temperature, $\mathrm{pH}$ of aqueous environment of body and acidic $\mathrm{pH}$ of stomach was simulated for drug delivery. For this purpose, the PBS of $\mathrm{pH}-2$ and hydrochloric acid with pH-1.2 were also used.

\section{RESULTS}

After studying the drug standard graph, data of the IR spectrum of net drug and produced microcapsules were also investigated. Then the optimum values of the materials were also obtained. Images obtained from microspheres were studied by scanning electron microscopy. In order to evaluate the effect of microcapsulasion processes, a comparison was carried out between the solubility of pure drug and coated drug. The drug release from the microcapsules produced in acidic environments and buffer will be also considered.

\section{Infrared spectroscopic studies}

Infrared spectroscopy study was taken to confirm that drug did not go under structural change problem in the process of microcapsulation. In this way, the pure and covered drug samples were analyzed by FTIR as the results confirmed that no change was occurred in the structure of the drug.

\section{Optimization processes}

Optimization of hydrocortisone acetate content

Changes in the initial drug concentration in the production process of microencapsulation affected the drug content. After production of microencapsulation, their drug content were 
measured. The obtained results can be seen in Figure 2. As can be seen by increasing the drug dose in the production of microspheres, the amount of drug absorbed in microencapsulation and thus the rate of drug release after consumption will increase. On the other hand by release of excessive drug (higher than optimal) inflation was seen in the microencapsulation and mass transfer resistance for drug delivery is occurred, which is undesirable and may cause rupture of microcapsules, that leads to sudden release of the drug.

Therefore, according to the structure of microcapsules, the highest mass content which does not make the mentioned problems for the microcapsules is considered as the optimum mass of drug, which is $0.06 \mathrm{~g}$.

\section{Optimization of ethyl cellulose content}

The amount of ethyl cellulose as the polymer used to produce microencapsulation is effective on the content of the drug. That is why different amounts of ethyl cellulose polymer used for preparation of microencapsulation were used by solvent evaporation technique and the amount of drug in a microencapsulation was also determined.
As shown in Figure 3, by increasing the amount of used polymer, the amount of drug absorbed in microencapsulation and thus the rate of drug release after consumption will also increase. The additional mass of ethyl cellulose polymer, (more than 0.15 grams), excessive cross connection occurs which causes production of dense polymer network with smaller pores. This resulted in a lower rate of drug coverage and increases the mass transfer resistance to drug delivery and thus reduces the load content of the drug. In addition, for the lower contents of polymer, because of the considerable reduction of monomers, the created polymer is not desirable for coverage of the drug and thickness of the wall, which will lead to decrease of capsulation efficiency. Also there will be the probability of microencapsulation discrete. Therefore, considering the points mentioned optimum mass of ethyl cellulose was about 0.15 grams.

\section{Optimization of dichloromethane content}

Another factor that can affect the efficiency of microcapsulation is the volume of used solvent. To evaluate the effect of solvent, the different volumes of dichloromethane were used for the production of microencapsulation and then the drug load of microencapsules were determined.
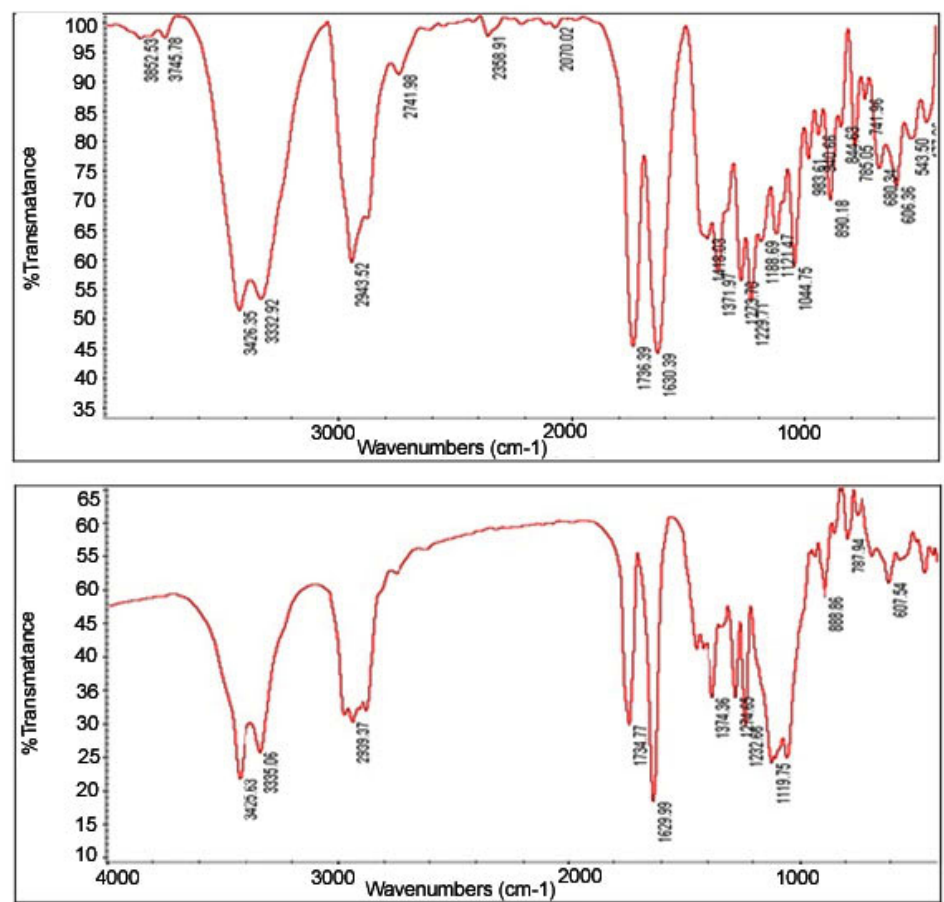

Fig. 1: Infrared spectra of a) pure drug hydrocortisone acetate, b) coated hydrocortisone 


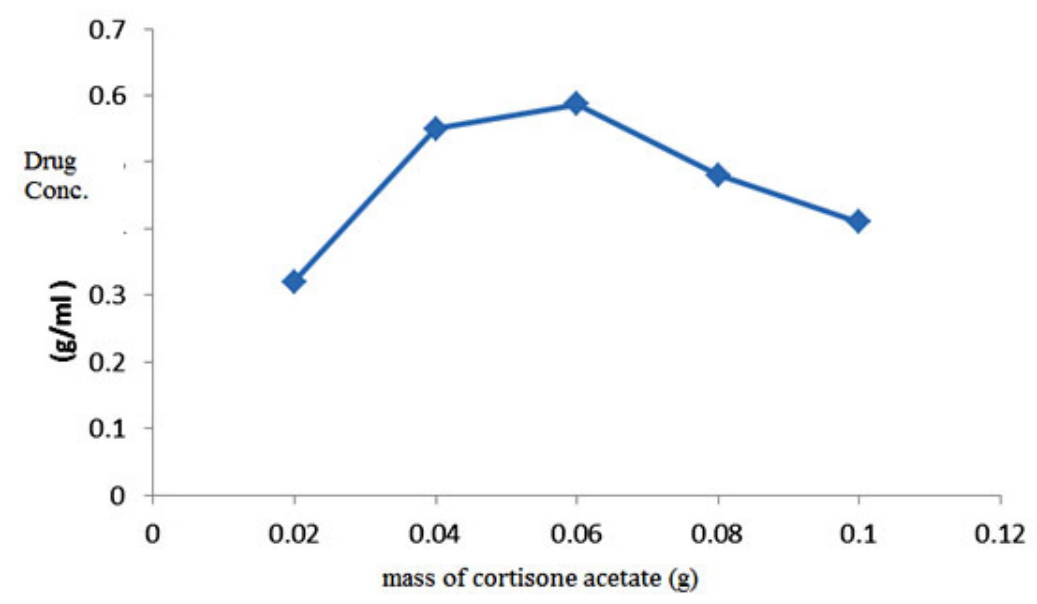

Fig. 2: Graph of drug concentration changes by mass of hydrocortisone acetate (different amounts of the drug's effect on the stability of microencapsulation)

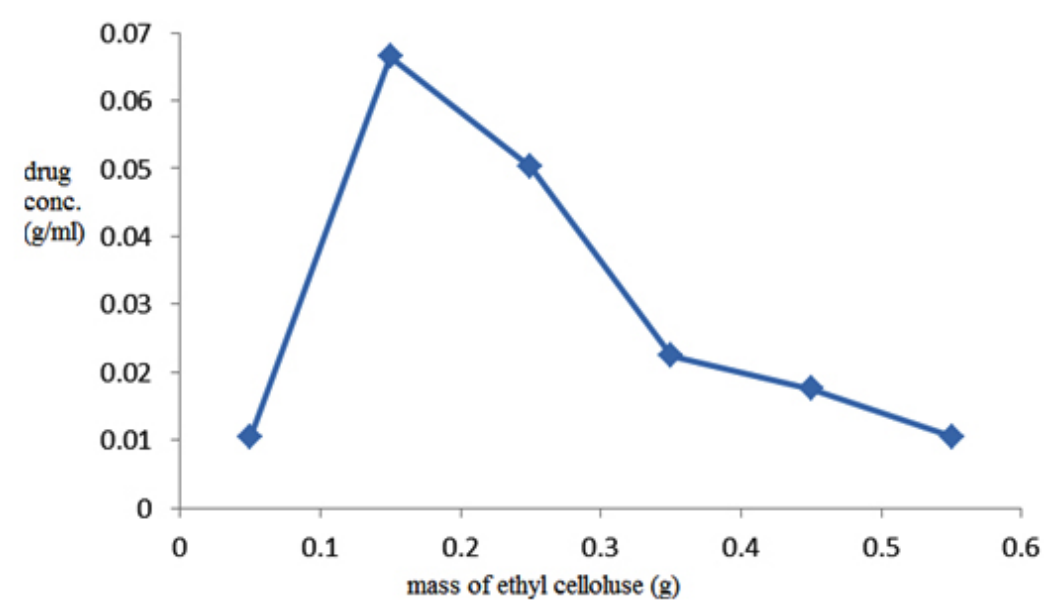

Fig. 3: Plot of drug concentration by mass of ethyl cellulose, (the effect of different amounts of polymer on stability of microencapsulation)

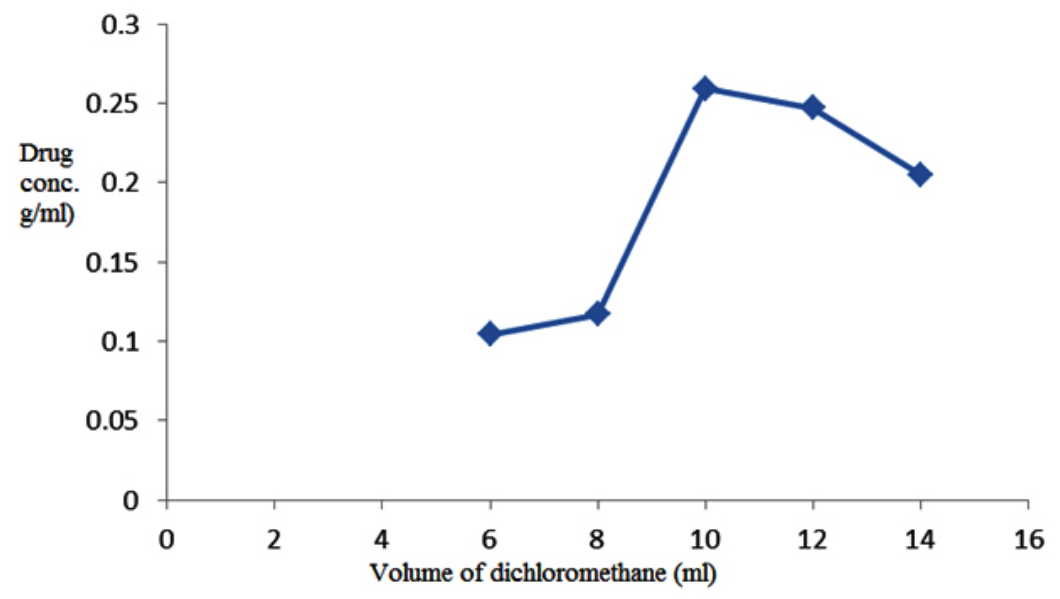

Fig. 4: Plot of change of drug concentration by volume of dichloromethane (Effect of different doses of solvent on the stability of microencapsulation) 
As shown in Figure 4, increasing the amount of solvent to the optimum value, the amount of drug absorbed in microencapsulation and thus the release of the drug after consumption will also increase. In amounts less than optimal level due to the increasing concentration of the polymer encapsulation efficiency reduces due to formation of dense and porous polymer coating. On the other hands, increase of solvent volume leads to decrease of viscosity and number of monomers in volume unit. This point also leads to production of undesirable polymeric networks with low strength and thickness. Due to the reasons discussed, the highest volume of dichloromethane that cause no problem for the microspheres is considered as the optimal volume of solvent, which is $10 \mathrm{ml}$.

\section{Optimization of polyvinyl alcohol content}

The amount of polyvinyl alcohol as an emulsifying agent for the preparation of microcapsules of hydrocortisone acetate is effective in the context of the drug. For this reason, various amounts of polyvinyl alcohol were used to prepare microencapsulation by solvent evaporation techniques and then the amount of drug in the microcapsules was determined. The results are presented in Figure (5).

As can be seen in Figure (5), increase of the amount of polyvinyl alcohol increases the amount of drug absorbed in microencapsules and consequently the amount of drug released after consumption. When the mass of PVA is more than the optimum amount $(0.5 \mathrm{~g})$, clots are formed which reduce the

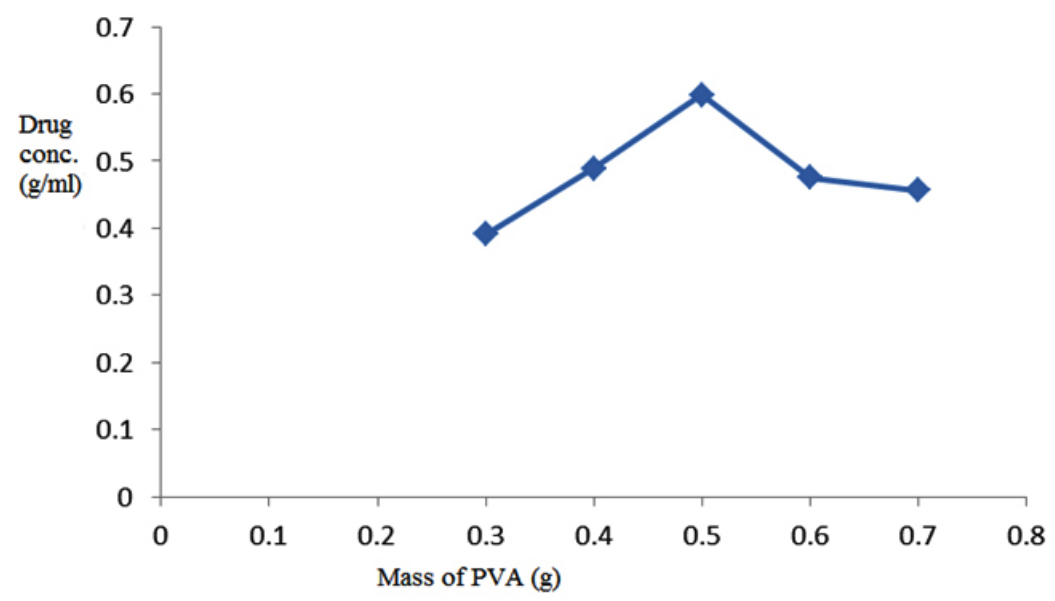

Fig. 5: The changes in drug concentration by mass of PVA (Effect of different doses of polyvinyl alcohol on stabilizing the microencapsulation)

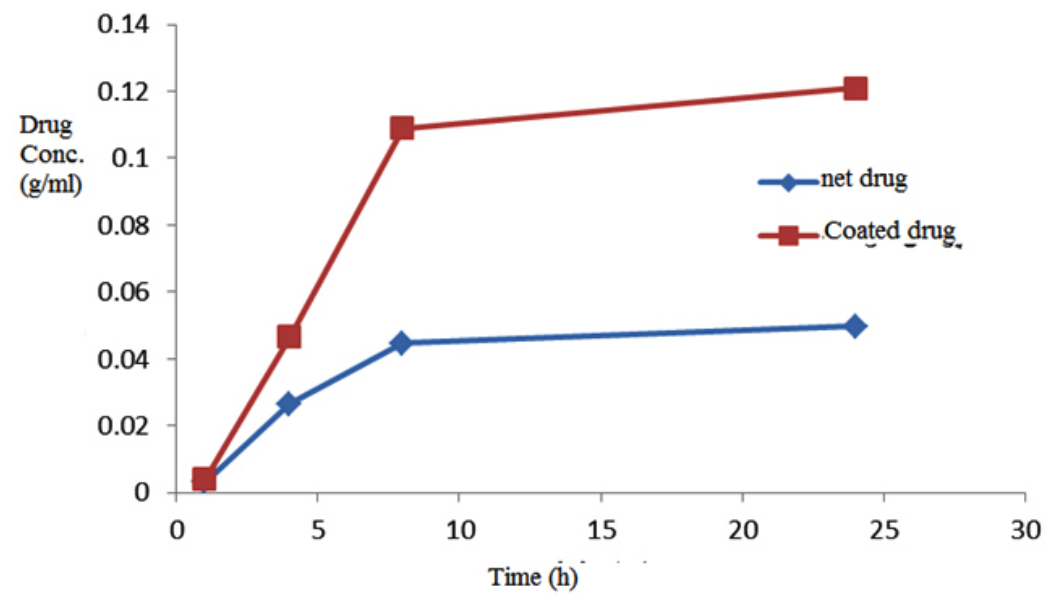

Fig. 6: changes in drug concentration over time (changes in net drug solubility and coated drug versus time) 
yields of polyvinyl alcohol for emulsion. As a result, efficiency of PVA for emulsification is reduced. Also in amounts less than optimal level, polyvinyl alcohol cannot be perform emulsification well and it is possible that two phases cannot be properly distributed in each other. It also can cause molecules to stick to each other and generate macromolecules instead of micromolecules.

\section{Studying the drug solubility}

In order to compare the aqueous solubility of pure and coated drugs, certain amount of the net drug, microcapsules containing a drug and drug-free microencapsules for $24 \mathrm{~h}$ were placed in distilled water in an incubator shaker at $37^{\circ} \mathrm{C}$. Figure 6 shows the solubility graph of pure and covered drug solubility in distilled water. Using solvent evaporation technique for microcapsulation of drug, solubility of coated drug was increased than the pure drug which is because of the presence of ethyl cellulose coatings around the drug. In fact, due to the properties of the polymer, it shows high performance in dispersion in aqueous media and as a result it is also distributed in the drug in the microcapsule. This leads to dissolution of drug in water and thereby increases the bioavailability. Additionally, because the polymer coating controlled the drug release does, the drug is released slowly and removes from the target environment less.

\section{Study of drug delivery}

Studying the drug release from the produced microcapsules was studied in buffer the acid media that simulate the body and stomach

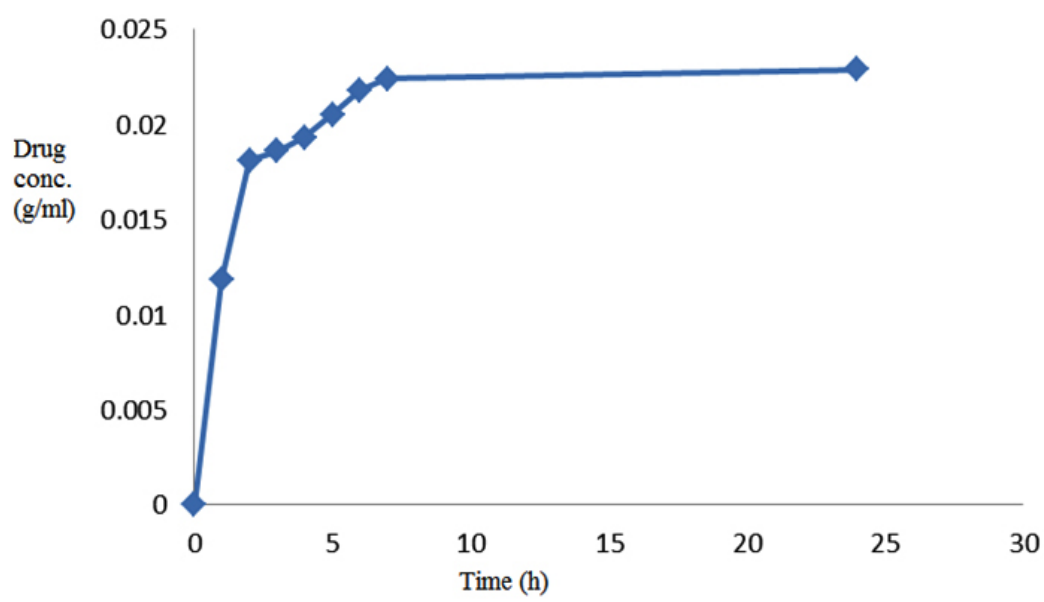

Fig. 7: Changes in drug concentration versus time (concentration of microcapsules for drug release in buffer solution)

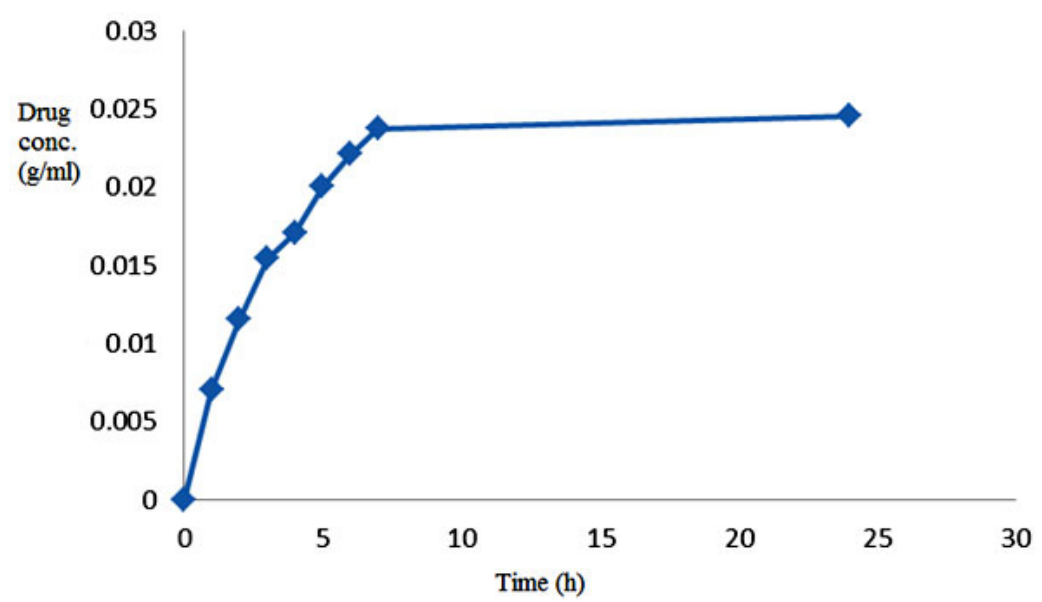

Fig. 8: Changes in drug concentration over time (changes of drug release from microcapsules delivery in an acid environment) 

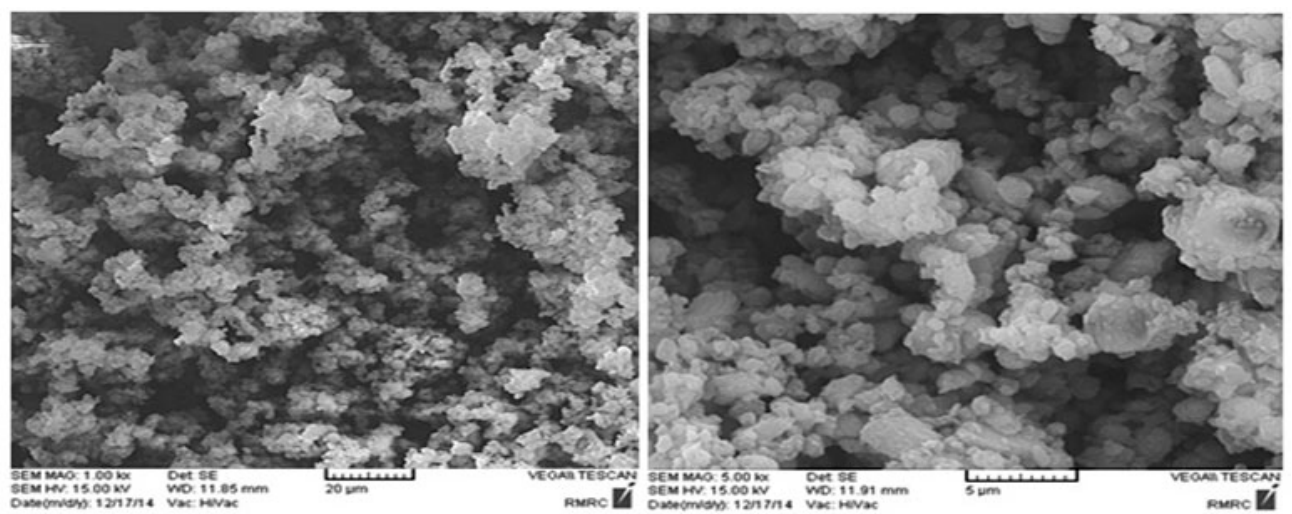

Fig. 9: Images obtained by the pure drug particles of hydrocortisone acetate
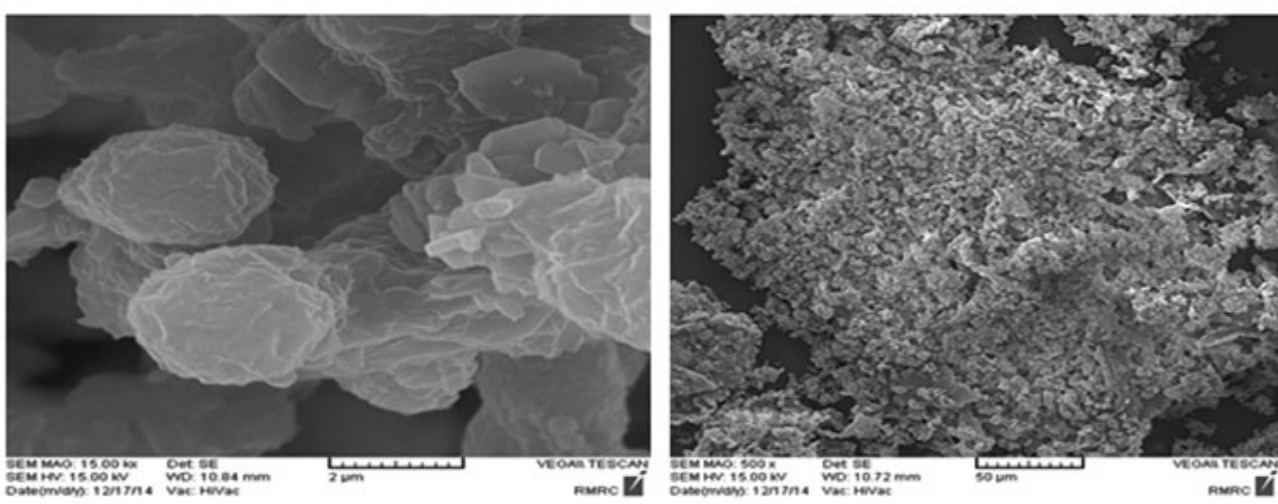

Fig.10: Images obtained from coated drug particles

environment. The results of this study have been reported separately. Drug release in buffer solution from hydrocortisone acetate microcapsules. In order to simulate the drug release in aqueous solution with a pH of 2.7, a certain amount of microcapsules containing drugs and drug-free microcapsules were placed in incubator shaker for $24 \mathrm{~h}$, maintaining the temperature of $37^{\circ} \mathrm{C}$.

Over the time, release of the drug from microcapsules was carried out. The release was first slow because the microcapsules require some time to penetrate into the aqueous environment and become prepared to release the drug. After a while, the release was faster. But at the end and final times, because of the reduction of drug load even after taking a long time, no considerable change was happened in release of the drug. Drug release in the acidic environment of hydrocortisone acetate microcapsules. In order to simulate drug delivery in the stomach environment with $\mathrm{pH}$ of
1.2 , the measured amounts of containing drugs and drug-free microcapsules were placed in an incubator shaker for $24 \mathrm{~h}$ under the temperature of $37^{\circ} \mathrm{C}$.

Release of drug from microcapsules was started over the time, which was slow at first. That was happened because of the time required by the microcapsules to penetrate to the aqueous media and become prepared for release. But after a while the release was increased and finally, in the final hours due to reduction in the content of the drug even over a longer period, a little change was established in release rate.

\section{The results obtained by scanning electron microscope \\ The scanning electron microscope} was used in order to determine the shape and size of the produced microcapsules using the technique of solvent evaporation. The results can 
be seen in Figures 9 and 10. As can be seen after microcapsulation of hydrocortisone acetate, spherical-shaped microcapsules are produced, the microspheres contained hydrocortisone acetate as the core drug and a polymer coating of ethyl cellulose.

\section{CONCLUSION}

In this study solvent evaporation technique was selected among the other techniques, as a subset of microcapsulation processes to increase the solubility and drug release of sample drug, hydrocortisone acetate. In this method microencapsulation were prepared using ethyl cellulose as the coating polymer. The amounts of materials used in the production of capsules, in order to achieve optimal conditions, were also optimized. Final optimal microcapsules drugs were prepared by $0.06 \mathrm{~g}$ of hydrocortisone acetate, $0.15 \mathrm{~g}$ of ethyl cellulose polymer, $10 \mathrm{ml}$ of dichloromethane solvent and $0.5 \mathrm{~g}$ of polyvinyl alcohol. Mass change of drug in formulation alters the content of the drug in pharmaceutical microspheres which decreases the output of capsulasion. If the amount of polymer is less than the threshold, the amount of monomers to form a polymer network will be negligible and therefore, the polymer is not favorable in terms of wall thickness for coating the drug. Changes in volumes of dichloromethane affects the polymer concentration and the concentration of drugs, as some problems that affect the crime rate due to changes in unit volume caused by the material have been mentioned. Excessive use of polyvinyl alcohol can cause blood clots and reduce its ability for desirable emulsification. The lack of this substance causes emulsification not be applied properly as the macromolecules are produced. Optimal Microcapsules produced in this study, were investigated in the aqueous condition of body besides the stomach in the case of drug release. The results showed that the drug hydrocortisone acetate in buffer with $\mathrm{pH}-7.2$ showed a better release then $\mathrm{pH}-1.2$. This is due to the hydrocortisone acetate tendency to dissolve in alkaline environments than acidic environments. FT-IR spectrophotometry was also used to confirm that no change was occurred in the chemical structure of the drug during the mentioned processes in solvent evaporation method. The results of the spectra of pure and drugs showed the accuracy of this issue. The results of SEM for determining the shape and size of the produced capsules represented a spherical microcapsules containing drugs of hydrocortisone acetate.

\section{REFERENCES}

1. Hoffman, S. J. Control Release, 2008, 132 , 153-163.

2. Hillery, A. M.; Anya, M. School of Pharmacy and Biomolecular Sciences university of Brighton, 2001, 215-333.

3. Qiu, Y.; Park, K. Adv. Drug .Deliv Rev. 2001, 53, 321-339.

4. Brasnjevic, I.; Steinbusch, HW.; Schmitz, C.; Martinez-Martinez, P. Prog. Neurobiol. 2009, 87, 212-251.

5. Sarkar, A. B.; Dudley, R.; Melethil, S.; Speidel, J.; Bhatt, G. M. Innovations in pharmacy, 2011, 2, 8.
6. Wischke, C.; Steven, P.; Deman, S. int. j. Pharm, 2008, 364, 298-327.

7. Ghandehari, H. Adv. Drug . Deliv. Rev. 2008, 60, 956.

8. Packhaeuser, C. B.; Schnieders, J.; Oster, C.G. Eur.J. Pharm .Biopharm. 2004, 58, 445-455.

9. Razzacki, S. Z.; Thwar, P.K.; Yang, M.; Ugaz, V.M.; Burns, M.A. Adv.Drug. Deliv. Rev. 2004, 56, 185-198

10. Yeo, Y.; Baek, N.; Park, K. Biotechnol. Bioprocess. Eng. 2001, 6, 213-230 